\title{
AOR
}

Selected Papers of \#AolR2021:

The 22nd Annual Conference of the

Association of Internet Researchers

Virtual Event / 13-16 Oct 2021

\section{AFFORDANCES IN DIGITAL DETOX AND PRODUCTIVITY APPS}

Faltin Karlsen

Kristiania University College

\section{Introduction}

The ubiquity of the smartphone has led to concerns about media becoming increasingly more intrusive and worries that that tech companies take advantage of our "easily distracted mind". This has also, alongside other commercial endeavors such as digital detox retreats and "dumb" mobiles, incited a booming app industry where digital detox and productivity apps are offered as tools to restrain smartphone use. As a relatively new phenomena this has so far received little attention from media scholars.

Intrusive media technology has, in general, received attention in media studies and related fields (Light 2014, Syvertsen 2020). Such studies usually revolve around the media user and give insight into various social and cognitive elements that play a role in the experience of being overwhelmed by media technology. The challenges of separating work from private life is one of many issues explored on this subject (Gregg 2011, Wajcman 2015, Karlsen and Ytre-Arne 2021). The technology itself, and what kind of affordances that trigger unwanted use, has received far less attention. To the extent affordances are discussed, it is usually in general terms, including how the smartphone may allow for micro-coordination (Thorhauge 2016), to generate a sense of "flow" (Ytre-Arne et al. 2020), or provide self-tracking practices (Lomborg et al. 2018, Lomborg and Fransen 2016). The aim of this study is to employ new empirical data and a conceptual framework to shed light on what kind of affordances that may stimulate and inhibit use of the smartphone.

\section{Methods, material and framework}

The analysis is based on an mapping of product descriptions of 70 digital detox and productivity apps retried from the App Store and Google Play. A selection of five apps were further subject to a design analysis conducted through "in the wild" testing over a 
period of 1-2 week for each app. The sample of apps were chosen for variety and cover a range of different affordances. The app Moment, for instance, rely mainly on inspirational quotes, tasks, and simple self-tracking affordances while the app Forest rely on a long range of feedback mechanisms, self-tracking features, and reward systems. The complete selection of apps are Hold, Forest, Cleverest, Moment, and Freedom.

Conceptually, the analysis distinguishes between two broad categories of affordances: undesign affordances, which comprise affordances that punish or inhibit certain types of use (Pierce 2012) and gamification affordances which, in general, reward the user or encourage certain user behavior (Deterding et al. 2011). These are often combined, meaning that a successful abstention session earns the player rewards in form of virtual resources, visual or textual praise or even real-world items such as discounts on wares. A discourse analysis of the production description is also conduced, aiming to see to what extent the affordances matches the overall aim with the apps, such as increasing productivity, mental wellbeing, a better lifestyle, or simply to spend less time on the smartphone.

\section{Preliminary findings}

A general finding in the sample of 70 apps is that they to a large extent are based on traditional self-help techniques including consciousness raising, creating (technical) hurdles, or productivity techniques such as pomodoro. Several of the apps also rely on affordances already existing in the operating systems such as turning off notification, using black/white interface, turning on flight mode, and moving apps to less accessible screen areas. In many cases they also emulate traditional analog time management techniques where a physical clock could just as easily be used. Only a smaller number of affordances are original, in the sense that they do not already exist in some form in the operating systems, or have an analog equivalent.

In the five apps subject to the design analysis, gamification affordances such as rewards, achievements, and ratings are generously used, while more relentless undesign affordances, including complete blocking of the smartphone, are uses to much lesser extent. Several apps are almost indistinguishable from ordinary casual smartphone games, such as farming games (HeyDay), idle games (Coocie Clicker), collecting games (Pokemon Go) and nursing/hatching games (Tamagotchi), implying that the apps use the same type of affordances that is driving engagement and intrusiveness in the apps they are trying to constrain. The frequent use of gamification design means that the apps, paradoxically, can direct more attention towards the smartphone. For instance, in order to earn gold needed to upgrade avatars or to develop other virtual items, the user has to return to the smartphone frequently. Being part of the attention economy, these apps compete for users' attention like any other 
app or media technology (Terronova 2012). While excessive use of gamification design may seem economical reasonable, it illustrates the innate paradox of trying to "beat technology with technology" (Syvertsen 2020: 90).

\section{References}

Deterding, S., Dixon, D., Khaled, R., \& Nacke, L. (2011, September). From game designelements to gamefulness: defining" gamification". In Proceedings of the 15th international academic MindTrek conference: Envisioning future media environments (pp. 9-15).

Gregg, M. (2018). Counterproductive: Time management in the knowledge economy. Duke University Press

Light, B. (2014). Disconnecting with Social Networking Sites (1. utg.). Palgrave Macmillan.

Karlsen, F, \& Ytre-Arne, B. (2021) Intrusive media and knowledge work: how knowledge workers negotiate digital media norms in the pursuit of focused work. Information, Communication \& Society (2021): 1-16.

Lomborg, S., Thylstrup, N. B., \& Schwartz, J. (2018). The temporal flows of selftracking: Checking in, moving on, staying hooked. New Media \& Society, 20(12), 45904607.

Pierce, James. "Undesigning technology: considering the negation of design by design." Proceedings of the SIGCHI Conference on Human Factors in Computing Systems. 2012.

Syvertsen, Trine. Digital Detox: The Politics of Disconnecting. Emerald Group Publishing, 2020

Terranova, T. (2012). Attention, economy and the brain. Culture Machine, 13.

Thorhauge, Anne Mette (2016) 'Balancing the flows: Cross-media Communication in an Everyday life Context', pp 55-73 i Sandvik, Kjetil mfl. (red.) The Media and the Mundane: Communication across media in Everyday Life. Gøteborg: Nordicom.

Ytre-Arne, Brita; Moe, Hallvard; Syvertsen, Trine and Karlsen, Faltin (2020). Temporal ambivalences in smartphone use: Conflicting flows, conflicting responsibilities, New media and Society 22(9) 1715-1732.

Wajcman, J. (2015). Pressed for time: The acceleration of life in digital capitalism. MIT Press 